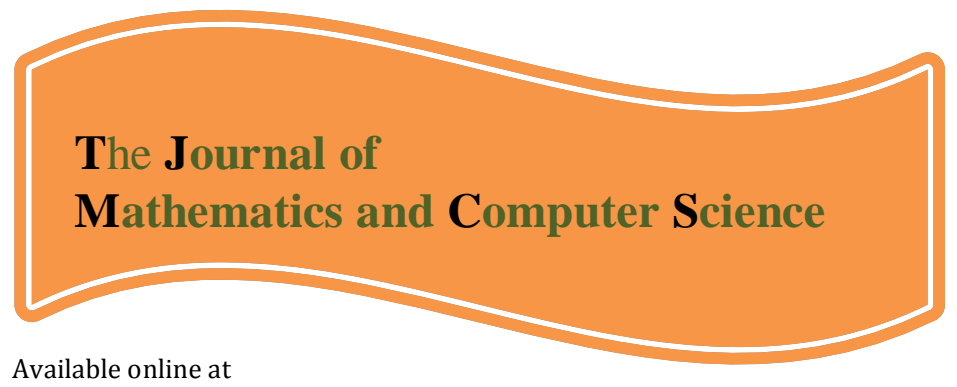

http://www.TJMCS.com

The Journal of Mathematics and Computer Science Vol .2 No.4 (2011) 580-587

\title{
A Novel Algorithm for Finding Equilibrium Strategy in Two Person Zero Sum Game with Fuzzy Strategy Sets and Payoffs
}

\author{
Seyyed Mohammad Reza Farshchi ${ }^{1,}$, Gelare Veisi ${ }^{2}$, Taghi Karimi ${ }^{3}$, Morteza Aghaei ${ }^{4}$ \\ ${ }^{1}$ Department of Artificial Intelligence, Islamic Azad University, Mashhad Branch, \\ Shiveex@gmail.Com \\ 2Department of Computer Engineering, Islamic Azad University, Mashhad Branch, \\ Gveisi@mshdiau.ac.ir \\ ${ }^{3}$ Department of Mathematics, Payam Noor University, Fariman Branch, \\ Taghi_karimi@pnu.ac.ir \\ 4Payam Noor University, Mashhad Branch, \\ Aghaei@pnu.ac.ir
}

Received: August 2010, Revised: November 2010

Online Publication: January 2011

\begin{abstract}
This paper investigates a two person zero sum matrix game in which the payoffs and strategy are characterized as random fuzzy variables. Using the operations of triangular fuzzy numbers, the fuzzy payoffs for all synthetic outcomes are calculated. Based on random fuzzy expected value operator, a random fuzzy expected minimax equilibrium strategy to the game is defined. After that, based on the constraints, the feasible strategy string sets of the players for multi conflict situations are constructed. Then an iterative algorithm based on random fuzzy simulation is designed to seek the minimax equilibrium strategy. Using a linear ranking function, the aggregation model can be solved by transforming it into a crisp bimatrix game. Then, the fuzzy synthetic aggregation model is established and solved by transforming it into a crisp bimatrix game. Finally, a military example is provided to illustrate the practicality and effectively of the model.
\end{abstract}

Keywords: Fuzzy game theory, two person game, equilibrium strategy, fuzzy payoff.

\footnotetext{
1,* Advanced Researcher in Imaging Science and Engineering Lab., Sadjad University of Mashhad.

${ }^{2}$ Associated Professor in Computer Engineering, Islamic Azad University, Mashhad Branch.

${ }^{3}$ Associated Professor in Payam Noor University, Fariman Branch.

${ }^{4}$ Payam Noor University, Mashhad Branch.
} 


\section{Introduction}

Since Von Neumann and Morgenstern's seminal work [5], game theory has been used extensively to analyze conflict and cooperative situations in economics, sociology, etc. One of the fundamental problems in the game theory is the two-player zero-sum game (with complete information), which has been discussed at length in the literature. However, as pointed out by Harsanyi [13], players in a real game often lack the information about the other players (or even his own) payoff functions. For instance, two competing firms are marketing the same new product. The goal of each firm is to attract as many customers as possible by choosing one from its marketing strategy set that includes TV advertiser, free samples etc. However, there is no past statistical evidence.

About the demand for the new product, and the effects of the firms marketing strategies on payoffs are very difficult to evaluate via probabilistic methods. In such situations, fuzzy set theory may offer an appropriate and powerful alternative, and make it possible to take human experiences, subjective judgment and intuitions into consideration. In such a framework, we may model the imprecise and vague payoffs as fuzzy variables, and indeed, many researchers have proposed games with fuzzy payoffs.

The fuzzy payoff games were developed by Aubin [1] and Butnariu [4] around 1980. Subsequently, many researchers have analyzed Nash equilibria in the framework of fuzzy set theory. For instance, Campos et al [5] [6] adopted some subjective ranking functions as the player's decision criteria, and analyzed the fuzzy payoff games. Nishizaki and Sakawa [2][7] defined equilibrium solutions via the degree of attainment of the fuzzy payoff and a fuzzy goal, and investigated their existence and other properties; Maeda [22][23] defined equilibrium solutions in terms of possibility measure and necessity measure, and analyzed their properties.

A fundamental problem of the classical game theory is that player makes decisions with crisp data. However, in a real life world, most games always take place in uncertain environments. There are two types of common uncertainties in the game randomness and fuzziness. Accordingly, there are two powerful theories, probability theory and possibility theory to deal with them. Employing the method of statistical mechanics, Berg and Engel [2], Ein-Dor and Kanter [3] discussed a two person zero sum matrix game with random payoffs. Based on the theory of fuzzy sets initiated by Zadeh [4], two-person zero sum matrix game with fuzzy payoffs was studied by Campos [5], who employed linear programming models to solve the fuzzy matrix game. As the extension of the idea of Campos [5], Nishizaki and Sakawa [6] discussed multi objective matrix game with fuzzy payoffs. Further more, Bector, Chandra and Vijay [7] introduced a dual for linear programming problems with fuzzy parameters to matrix games with fuzzy payoffs. Vijay, Chandra and Bector [8] provided a solution procedure for solving two person zero-sum matrix games with fuzzy goals and fuzzy payoffs.

When game theory is applied to real world problems, sometimes the payoffs contain both randomness and

fuzziness. In such a case, randomness and fuzziness are required to be considered simultaneously. Kwakernaak [9][10], first introduced the notion of fuzzy random variables, then this concept was developed by several researchers such as Puri and Ralescu [11], and Kruse and Meyer [12]. Roughly speaking, a fuzzy random variable defined by Kwakernaak [9], [10] is a measurable function from a probability space to a collection of fuzzy numbers, and its expected value is described as a fuzzy number. From the viewpoint that fuzzy random variable is a measurable function from a probability space to a collection of fuzzy numbers, Yoshida [13] discussed a stopping game for a sequence of fuzzy random variables and gave a saddle point. Another concept of the fuzzy random variable defined by Liu and Liu [14] is a measurable function from a probability space to a collection of fuzzy variables and its expected value was defined as a scalar value. Xu, Zhao and Ning [15] discussed a two person zero sum matrix game with fuzzy random payoffs, in which the notion of fuzzy random variable is proposed by Liu and Liu [14].

When a player in a game plays a strategy to the other player's actual strategy choices, the payoffs that the player receives may be probability distribution that is completely known except for the values of one or more parameters. For example, it is widely accepted that the amount of net cash flow is normally 
distribution variable $N(\mu, \sigma)$, but the value of $\mu, \sigma$ cannot be exactly assessed due to inaccuracy of information. In such situations, we need to take into account probability distribution with fuzzy parameters and deal with the payoffs as random fuzzy variables. In contrast with fuzzy random variables, Liu [16] defined a random fuzzy variable from a possibility space to a collection of random variables.

In this paper, we discuss a two person zero sum matrix game with random fuzzy payoffs, in which the notion of random fuzzy variable is proposed by Liu [16]. A random fuzzy expected minimax equilibrium strategy is provided, and an iterative algorithm based on random fuzzy simulation is designed to find the expected minimax equilibrium strategy. Finally, a numerical example is provided to illustrate this idea.

\section{Preliminaries}

Let $\theta$ be a nonempty set, $P(\theta)$ the power set of $\theta$, and Pos a possibility measure, then the triplet $(\theta, P(\theta), P o s)$ is called a possibility space. Based on the possibility measure Pos, the credibility $\mathrm{Cr}$ of a fuzzy event was defined.

Definition 1: (Liu and Liu [17]) Let $(\theta, P(\theta), P o s)$ be a possibility space, and $A$ is a set in $P(\theta)$, then the

Credibility measure of $\mathrm{A}$ is defined by:

$$
\operatorname{Cr}\{A\}=\frac{1}{2}\left(\operatorname{Pos}\{A\}+1-\operatorname{Pos}\left\{A^{c}\right\}\right)
$$

Where $A^{c}$ is the complement of $A$.

Definition 2: A fuzzy variable $\xi$ is a function defined on a possibility space $(\theta, P(\theta), P o s)$ to the set of real

numbers, and its membership function is derived from the possibility measure by:

$$
\mu(x)=\operatorname{Pos}\{\theta \in \Theta \mid \xi(\theta)=x\}, x \in \mathfrak{R}
$$

Definition 3: (Liu and Liu [17]) Let $\xi$ be a fuzzy variable on the possibility space $(\theta, P(\theta), P o s)$, the expected value $\mathrm{E}[\xi]$ is defined by:

$$
E[\xi]=\int_{0}^{+\infty} C r\{\xi \geq r\} d r-\int_{0}^{+\infty} C r\{\xi \leq r\} d r
$$

Provided that at least one of the two integrals is finite.

Definition 4: An n-dimensional fuzzy vector is defined as a function from a probability space $(\Omega, A, \operatorname{Pr})$ to the set of $\mathrm{n}$-dimensional real vectors.

the

Example 1: In many statistics problems, the probability distribution is completely known except for

values of one or more parameters. For example, it might be known that the lifetime $\xi$ of a modern engine is an exponentially distributed variable with an unknown mean $\lambda$ :

$$
\phi(x)= \begin{cases}\frac{1}{\lambda} e^{-x / \lambda}, & \text { if } 0 \leq x<\infty \\ 0 & \text { otherwise }\end{cases}
$$


Usually, there is some relevant information in practice. It is thus possible to specify an interval in which the value of $\lambda$ is likely to lie, or to give an approximate estimate of the value of $\lambda$. It is typically not possible to determine the value of $\lambda$ exactly. If the value of $\lambda$ is provided as a fuzzy variable defined on the possibility spaces $(\theta, P(\theta), P o s)$ then $\xi$ is a random fuzzy variable.

Theorem 1: Assume that $\xi$ is a random fuzzy variable, then the probability $\operatorname{Pr}\{\xi(\theta) \in B\}$ is a fuzzy variable for any Borel set $B$ of $R$.

Theorem 2: Let $\xi$ be a random fuzzy variable. If the expected value $E[\xi(\theta)]$ is finite for each $\theta$ then $\mathrm{E}[\xi(\theta)]$ is a fuzzy variable.

Definition 6: An n-dimensional random fuzzy vector is a function from the possibility space $(\theta, P(\theta), P o s)$ to the set of $\mathrm{n}$-dimensional random vectors.

Theorem 3: The vector $\left(\xi_{1}, \xi_{2}, \ldots, \xi_{n}\right)$ is a random fuzzy vector if and only if $\xi_{1}, \xi_{2}, \ldots, \xi_{n}$ are random fuzzy variables.

Theorem 4: Let $\xi$ be an n-dimensional random fuzzy vector, and $f: R^{n} \rightarrow R$ is a Borel measurable function, then $f(\xi)$ is a random fuzzy variable.

Definition 7: (Liu and Liu[18]) Let $\xi$ be a random fuzzy variable, then the expected value $\mathrm{E}[\xi]$ is defined by:

$$
E[\xi]=\int_{0}^{+\infty} \operatorname{Cr}\{\theta \in \Theta \mid E[\xi(\theta)] \geq r\} d r-\int_{0}^{+\infty} \operatorname{Cr}\{\theta \in \Theta \mid E[\xi(\theta)] \leq r\} d r
$$

Provided that at least one of the two integrals is finite.

\section{Two Person Zero Sum Matrix Game}

In this section, we discuss a two person zero sum matrix game in which the payoffs are characterized as fuzzy random variables, and then we define a fuzzy random expected minimax equilibrium strategy to the game.

Let $\stackrel{\Delta}{I}=\{1,2, \ldots, m\}$ denotes a set of pure strategies of player I and $J \stackrel{\Delta}{=}\{1,2, \ldots, n\}$ denotes a set of pure strategies of player II. Mixed strategies of players I and II are represented by weights to pure strategies of them, i.e.,

$$
S^{m}=\left\{\left(x_{1}, x_{2}, \ldots, x_{m}\right)^{T} \in \mathfrak{R}_{+}^{m} \mid \sum_{i \in I} x_{i}=1\right\}
$$

denotes strategy space for player I, and,

$$
S^{n}=\left\{\left(y_{1}, y_{2}, \ldots, y_{n}\right)^{T} \in \mathfrak{R}_{+}^{n} \mid \sum_{j \in J} y_{j}=1\right\}
$$

denotes strategy space for player II, where $\mathfrak{R}_{+}^{m}=\left\{a \in \mathfrak{R}^{m} \mid a_{i} \geq 0, i=1, \ldots, m\right\}$ and $x^{T}$ is the transposition of $x$. 
Let random fuzzy variable $\xi_{i j}$ represent the payoffs that player I receives or player II loses when player I plays the pure strategy $i$ and player II plays the pure strategy $j$, then a two person zero sum game is represented by random fuzzy payoffs matrix such,

$$
\eta=\left[\begin{array}{cccc}
\xi_{11} & \xi_{12} & \ldots & \xi_{1 n} \\
\xi_{21} & \xi_{22} & \ldots & \xi_{2 n} \\
\cdot & . & \ldots & . \\
\xi_{m 1} & \xi_{m 2} & \ldots & \xi_{m n}
\end{array}\right]
$$

When the mixed strategies $\mathrm{x}$ and $\mathrm{y}$ are chosen by player I and player II, respectively, then the random fuzzy payoffs of player I are:

$$
x^{T} \eta y=\sum_{j=1}^{n} \sum_{i=1}^{m} \xi_{i j} x_{i} y_{j}
$$

and the random fuzzy payoffs of player II are: $-x^{T} \eta y$.

Since the payoffs are characterized as random fuzzy variables, the player cannot predict how many the payoffs will actually be when he chooses a strategy. It is a nature idea for the player to maximize his or minimize the opponents random fuzzy expected payoffs. The problem for the player is to choose the strategy so that the random fuzzy expected payoffs achieves the optimal values. So based on random fuzzy expected value operator, we give the following minimax equilibrium strategy.

Definition 8: Let $\xi_{i j}(i=1,2, \ldots, m, j=1,2, \ldots, n)$ be random fuzzy variables with finite expected values, Then $\left(x^{*}, y^{*}\right) \in S^{m} \times S^{n}$ is called a random fuzzy expected minimax equilibrium strategy to the game if:

$$
E\left[x^{T} \eta y\right] \leq E\left[x^{* T} \eta y^{*}\right] \leq E\left[x^{* T} \eta y\right]
$$

Where $\eta$ is defined by (5).

\section{Iterative Algorithm based on Random Fuzzy Simulation}

Stochastic simulation has been applied to numerous areas, and is defined as a technique of performing sampling experiments on the models of stochastic systems. Let $\xi$ be an n-dimensional random vector defined on the probability space $(\Omega, A, \operatorname{Pr})$ and $f: R^{n} \rightarrow R$ be a measurable function. Then $\mathrm{f}(\xi)$ is a random variable. The expect value of $\mathrm{E}[\mathrm{f}(\xi)]$ can be evaluated by the following algorithm.

\section{Algorithm 1: Finding Expected Value}

Step 1. Set $\mathrm{L}=0$.

Step 2. Generate $\omega$ from $\Omega$ according to the probability measure Pr.

Step 3. $L \leftarrow L+f(\xi(\omega))$.

Step 4. Repeat the second and third steps $\mathrm{N}$ times, where $\mathrm{N}$ is a sufficiently large number.

Step 5. $E[f(\xi)]=L / N$.

Although simulation is an imprecise technique which provides only statistical estimates rather than exact results and is also a slow and costly way to study problems, it is indeed a powerful tool dealing with complex problems without analytic techniques. When payoffs are characterized as random fuzzy variables, traditional analytical Solution methods are not applicable to find a random fuzzy expected 
minimax equilibrium strategy. The random fuzzy simulation technique proposed by [16] is an effective tool to evaluate the expected value of random fuzzy payoffs. In this section, we propose an algorithm, in which random fuzzy simulation is employed to estimate the expected value, and iterative technique is used to seek the minimax equilibrium strategy.

Let $\xi$ be an n-dimensional random fuzzy vector, The expect value of $E[f(\xi)]$ can be estimated by the following procedure.

Step 1. Set $\mathrm{E}=0$.

Step 2. Randomly sample $\theta_{k}$ from $\Theta$ such that $\operatorname{Pos}\left\{\theta_{k}\right\} \geq \varepsilon, k=1,2, \ldots, N$, where $\varepsilon$ is a sufficiently small number and $\mathrm{N}$ is a sufficiently large number.

Step 3. Let $a=\min _{1 \leq k \leq n} E\left[f\left(\xi\left(\theta_{k}\right)\right)\right], k=1,2, \ldots, N$, can be obtained by random simulation above.

Step 4.

If $r \geq 0, E \leftarrow E+C r\{\theta \in \Theta \mid E[f(\xi(\theta))] \geq r\}$, where the credibility $\operatorname{Cr}\{\theta \in \Theta \mid E[f(\xi(\theta))] \geq r\}$ can

be estimated by $\frac{1}{2} \max _{1 \leq K \leq N}\left\{V_{k} \mid E[f(\xi(\theta))] \geq r\right\}+\frac{1}{2} \min _{1 \leq K \leq N}\left\{1-V_{k} \mid E[f(\xi(\theta))]<r\right\}$.

Step 5.

if $r<0, E \leftarrow E-C r\{\theta \in \Theta \mid E[f(\xi(\theta))] \leq r\}$, where the credibility $\operatorname{Cr}\{\theta \in \Theta \mid E[f(\xi(\theta))] \leq r\}$ can

be estimated by $\frac{1}{2} \max _{1 \leq K \leq N}\left\{V_{k} \mid E[f(\xi(\theta))] \leq r\right\}+\frac{1}{2} \min _{1 \leq K \leq N}\left\{1-V_{k} \mid E[f(\xi(\theta))]>r\right\}$.

Step 6. Repeat the fourth to sixth steps for $\mathrm{N}$ times.

Step 7. $E[f(\xi)]=a \vee 0+b \wedge 0+E \cdot(b-a) / N$.

Now we embed random fuzzy simulation into an iterative algorithm to find the random fuzzy expected minimax Equilibrium strategy. The procedure is summarized as follows:

Step 1. Firstly, let $x_{i 1}$ be a pure strategy selected randomly from the set of pure strategies $\{1,2, \cdots, \mathrm{m}\}$ by player I, and $y_{j 1}$ a pure strategy selected randomly from the set of pure strategies $\{1,2, \cdots, \mathrm{n}\}$ by player II.

Step 2. After the $t$ th game, the pure strategies selected by player I are $x_{i 1}, \ldots, x_{i t}$ while the pure strategies selected by player II are $y_{j 1}, \ldots, y_{j t}$. In the $(\mathrm{t}+1)$ th game, the pure strategy $x_{i t+1}$ selected by player I should satisfy the condition:

$$
\sum_{k=1}^{t} E\left[\xi_{i t+1 j k}\right]=\max _{1 \leq i \leq m} \sum_{k=1}^{t} E\left[\xi_{i j k}\right]
$$

The pure strategy $y_{j t+1}$ selected by player II should satisfy the condition:

$$
\sum_{k=1}^{t} E\left[\xi_{i k j t+1}\right]=\min _{1 \leq j \leq n} \sum_{k=1}^{t} E\left[\xi_{i k j}\right]
$$

Where $E\left[\xi_{i j}\right]$ can be estimated by random fuzzy simulation.

Step 3. Return to Step 2 with $t=t+1$ until the iteration number is $\mathrm{N}$, here $\mathrm{N}$ is a predetermined number of iterations.

Step 4. Let $x^{*}=\left\{x_{1}^{*}, x_{2}^{*}, \ldots, x_{m}^{*}\right\}$ where $x_{i}^{*}$ is the ratio of times of the pure strategy $i$ selected by player I in the above mentioned steps to $\mathrm{N}, i=1,2, \ldots, m$. Let $y^{*}=\left\{y_{1}^{*}, y_{2}^{*}, \ldots, y_{n}^{*}\right\}$ where $y_{j}^{*}$ is the ratio of times of the pure strategy j selected by player II in the above-mentioned steps to $\mathrm{N}, j=1,2, \ldots, n$. 
Step 5. Estimate $E\left[x^{* T} \eta y^{*}\right]$ by random fuzzy simulation, then display the random fuzzy expected minimax equilibrium strategy $\left(x^{*}, y^{*}\right)$ and $E\left[x^{* T} \eta y^{*}\right]$.

\section{Numerical Example}

An example is given to illustrate the idea of the matrix game with random fuzzy payoffs and the effectiveness of the algorithm provided in Section 4. Suppose we have:

$$
\eta=\left[\begin{array}{ll}
\xi_{11} & \xi_{12} \\
\xi_{21} & \xi_{22}
\end{array}\right]
$$

Where random fuzzy variables $\xi_{i j}(i=1,2, j=1,2)$ are characterized as:

$$
\begin{aligned}
& \xi_{11} \sim \exp \left(\lambda_{11}\right), \text { where } \lambda_{11}=(0,1,2,3) . \\
& \xi_{12} \sim \exp \left(\lambda_{12}\right), \text { where } \lambda_{11}=(2,3,4,5) . \\
& \xi_{21} \sim \exp \left(\lambda_{21}\right), \text { where } \lambda_{11}=(3,4,5,6) . \\
& \xi_{22} \sim \exp \left(\lambda_{22}\right), \text { where } \lambda_{11}=(1,2,3,4) .
\end{aligned}
$$

By 100 iterations (3000 sample points in random fuzzy simulation), the fuzzy random expected minimax equilibrium strategy is:

$$
\left(\left(x_{1}^{*}, x_{2}^{*}\right),\left(y_{1}^{*}, y_{2}^{*}\right)\right)=((0.4998,0.5002),(0.2500,0.7500)), \text { with } E\left[x^{* T} \eta y^{*}\right]=3.0484 \text {. }
$$

\section{Conclusion}

In this paper, a two person zero sum matrix game with random fuzzy payoffs is studied. Employing random fuzzy variables to describe the payoffs of the game, we present a random fuzzy expected minimax equilibrium strategy. An algorithm based on random fuzzy simulation is designed to seek the minimax equilibrium strategy.

\section{References}

[1] Von Neumann, J., and Morgenstern, 0., "Theory of Games and Economic Behavior", Wiley, 1944.

[2] Berg, J., and Engel, A., "Matrix games, mixed strategies, and statistical mechanics", Physical Review Letters, Vol. 81, pp. 4999-5002, 1998.

[3] Ein-Dor, L., and Kanter, I., "Matrix games with non uniform payoff distributions", Physical Letters, Vol. 302, pp. 80-88, 2001.

[4] Zadeh, L., "Fuzzy Sets", Information and Control, Vol. 8, pp. 338-353, 1965.

[5] Campos, L., "Fuzzy linear programming models to solve fuzzy matrix games", Fuzzy Sets and Systems, Vol. 32, pp. 275-289, 1989.

[6] Nishizaki, I., and Sakawa, M., "Fuzzy and Multi objective Games for Conflict Resolution", Fuzzy Sets and Systems, 2001.

[7] Bector, C., Chandra, S., and Vijay, V., "Duality in linear programming with fuzzy parameters and matrix games with fuzzy payoffs", Fuzzy Sets and Systems, Vol. 146, pp. 253-69, 2004. 
[8] Vijay, V., Chandra, S., and Bector, C., "Matrix games with fuzzy goals and fuzzy payoffs", Information Systems, Vol. 33, pp. 425-429, 2005.

[9] Kwakernaak, H., "Fuzzy random variables-I", Information Sciences, Vol. 15, pp. 1-29, 1978.

[10] Kwakernaak, H., "Fuzzy random variables-II", Information Sciences, Vol. 17, pp. 153-178, 1979.

[11] Puri, M., and Ralescu, D., "Fuzzy random variables", Journal of Mathematical Analysis and Applications, Vol. 114, pp. 409-422, 1986.

[12] Liu, L., and Liu, B., "Expected value operator of random fuzzy variable and random fuzzy expected value models", International Journal of Uncertainty, Fuzziness \& Knowledge-Based Systems, Vol. 11, No. 2, pp. 195-215, 2003.

[13] Yoshida, Y., "A stopping game in a stochastic and fuzzy environment", Mathematical and Computer Modeling, Vol. 30, pp. 147-158, 1999.

[14] Liu, L., and Liu, B., "Fuzzy random variables: a scalar expected value", Fuzzy Optimization and Decision Making, vol.2, No. 2, pp. 143-160, 2003.

[15] Xu, L., Zhao, R., and Ning, Y., "Two person zero sum matrix game with fuzzy random payoffs", Lecture Notes in Artificial Intelligence, Vol. 4114, pp. 809-818, 2006.

[16] Liu, B., "Theory and Practice of Uncertain Programming", Heidelberg, Physica-Verlag, 2002. 